\title{
New Experimental Approach to the Detection of Positron Annihilation in Flight Process
}

\author{
J. DRYZEK \\ Institute of Nuclear Physics PAN \\ Radzikowskiego 152, 31-342 Kraków, Poland \\ and \\ Institute of Physics, University of Zielona Góra \\ Prof. Szafrana 4a, 65-516 Zielona Góra, Poland
}

\begin{abstract}
Positron annihilation in flight, when mainly positron contributes to the energy and momentum of the annihilating pair, is rarely observed in condensed matter due to its small probability. Additionally, this process does not manifest itself by any characteristic features, which would help in observation. Hence, our knowledge about that process is poor. In the paper we propose the new experimental method which allows us to detect this process. We applied the conventional positron lifetime spectrometer, which after changing photon energy ranges and layout of detectors was suitable for that detection. The first measurements allow us to estimate that in pure aluminum almost $4.76 \%$ of all implanted positrons from isotope source ${ }^{22} \mathrm{Na}$ annihilate in flight.
\end{abstract}

PACS numbers: 78.70.Bj, 07.85.--m

\section{Introduction}

It is well known that an energetic positron implanted into matter rapidly slows down to the thermal energy in a time smaller than $10 \mathrm{ps}$. Then, within a time of a few hundred ps it randomly walks and after finding an electron finishes its life, due to the annihilation process. The emission of two quanta in almost opposite directions with an energy close to $511 \mathrm{keV}$ with a probability of about $99.8 \%$ is the major annihilation channel. Mainly, it is because the cross section for two-quanta annihilation in low-energy approximation is a reciprocal function of the positron velocity [1]. (Other possible annihilation channels, like the emission of a single photon or more than two photons, are neglected in our considerations.) The fact that the positron annihilates with much lower energy than an electron is used in positron annihilation spectroscopy, which provides unique information, e.g. 
about the electron momentum distribution in single crystal solids and crystalline defects [2]. Annihilation of thermal positrons is called annihilation at rest (AaR). However, annihilation with non-thermal, much higher energies is not excluded hereafter referred to as annihilation in flight (AiF). Nevertheless, this is a rare process; detailed calculations point out that few percent of positron emitted from the isotope sources can annihilate in flight [3]. The AiF process is willingly studied using a rather energetic positron beam, e.g. a search for anomalies in electronpositron scattering near the $\mathrm{Z}^{0}$ mass in $\mathrm{GeV}$ range [4]. Also, a low energy i.e. $71.6 \mathrm{keV}$, positron beam was used for detection of the AiF process [5].

The AiF process in condensed matter is poorly examined due to lack of experimental method, which in efficient way would allow us to observe it. The use of coincidence Doppler broadening spectrometer, which consists of two germanium detectors, seems to be a proper tool for that purpose [6]. In this experiment the energy of two photons in coincidence is measured and stored in the 2D diagram. In that diagram this process reveals itself as a very weak hyperbolic curve hidden in the background. This measurement is rather difficult because requires very efficient reduction of background from the environment. In the paper we intend to present another method which seems to be much simpler and less costly because applies the conventional positron lifetime spectrometer.

\section{The experimental setup}

During the AiF process the energy and momentum of a positron contributes mainly to the energy and momentum of the annihilating pair. This causes that the angle between the directions of emitted photons is much smaller than $180^{\circ}$ (Fig. 1b) as it is in AaR (Fig. 1a). Additionally, the energy of the two photons fulfills the following hyperbolic relation [6]:

$$
\frac{1}{E_{1}}+\frac{1}{E_{2}}=\frac{1-\cos (\theta)}{2 m_{0} c^{2}},
$$

where $m_{0}$ is the electron mass, $c$ - the speed of light, and $\theta$ is the angle between the direction of two photons. This is a main difficulty in detection of the AiF process because even for selected $\theta$ the energy of emitted photon varies between certain minimal and maximal values (see Eqs. (7a) and (7b) in Ref. [6]). Nevertheless, because the two photons are emitted simultaneously from the place where the annihilation takes place thus for detection one can use the setup commonly applied for the measurement of the positron lifetime. In the lifetime spectrometer, one detector branch is dedicated for detection of gamma ray of energy $1.27 \mathrm{MeV}$, being a signal of positron birth and the second one for detection of an annihilation photon of energy $0.511 \mathrm{MeV}$ which indicates the annihilation process. In the proposed setup both detection branches are applied for detection of photons of energy between the minimal and maximal values (for the positrons emitted from ${ }^{22} \mathrm{Na}$ it is ranged between $300 \mathrm{keV}$ and $1200 \mathrm{keV}$ ). Additionally, the positron emitter should be located above the line, which connects the centers of two detectors to ensure 

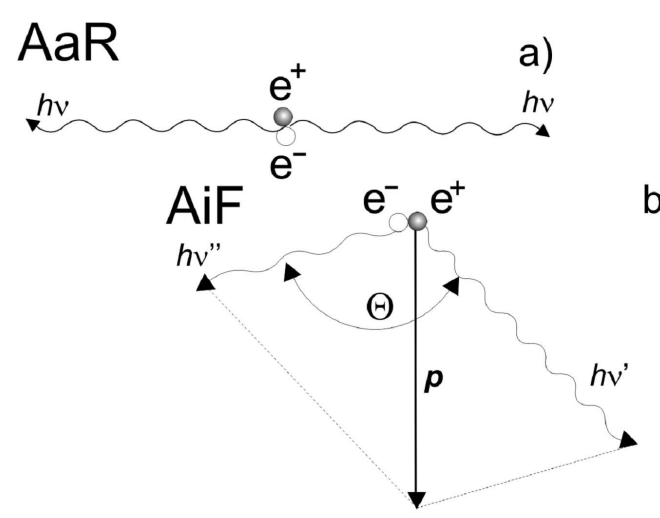

b)

Fig. 1. A schematic picture of annihilation (a) at rest and (b) annihilation in flight. For annihilation at rest the angle between the photons' momentum is close to $180^{\circ}$ but in flight $\theta<180^{\circ}$.

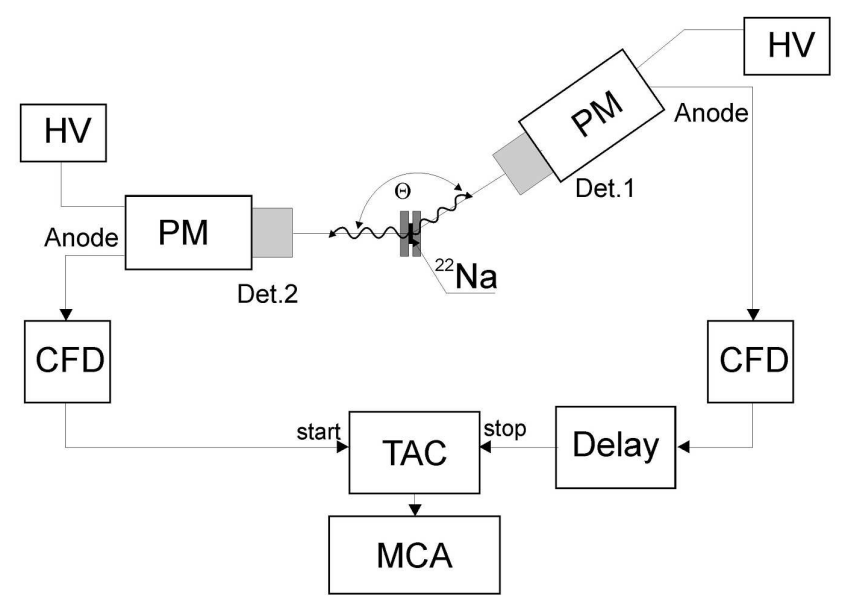

Fig. 2. The experimental setup for measuring of the prompt peak which manifests the time difference when two photons emitted in the positron-electron annihilation process travel to the two detectors.

the certain direction of traveling photons (Fig. 2). This is the base of the new experimental setup, which should allows us to detect the AiF process.

The emitted photons were detected by two BaF2 crystal scintillator detectors which were glued to the photomultipliers (Philips XP2020Q). The crystal was 1 inch in diameter and 1 inch in thickness. The fast pulse from the anode of the photomultiplier whose amplitude was also proportional to the energy of the absorbed photons was sent to the differential constant fraction discriminator (CFD) (ORTEC 583) (Fig. 2). This module allowed us to select the energy range of accepted input pulses and generated a NIM (Nuclear Instrumentation Module standard) fast negative output pulse. We chose an energy range between $300 \mathrm{keV}$ 
and $1200 \mathrm{keV}$ for the spectrum of emitted photons seen by the two detectors. The output pulse was dispatched to the time-to-amplitude converter module (ORTEC 566 ) which generated a pulse with an amplitude proportional to the time difference between the input pulses which occurred at the "start" and "stop" inputs. In the setup, two branches were fixed for detection of two annihilation photons. In one branch a delay module (ORTEC 425A) was mounted. This branch supplied pulses into the "stop" input of the time-to-amplitude converter. The output pulses from this converter are stored in a multichannel analyzer based on the ADC of Canberra $8 \mathrm{~K}$. One should mention that this is a typical setup of positron lifetime spectrometer which allows one to measure the positron lifetime. Only the difference is the energy range fixed in two branches.

\section{The results and discussion}

In our experiment we employed a standard ${ }^{22} \mathrm{Na}$ source such as is generally used in positron annihilation spectroscopy. The isotope of activity of $c a .50 \mu \mathrm{Ci}$ was enveloped in a $7 \mu \mathrm{m}$ thick kapton foil. This source was sandwiched by two pure aluminium plates of size $2 \mathrm{~cm} \times 2 \mathrm{~cm}$ and $2 \mathrm{~mm}$ thick. This set ensures to stop all emitted positrons in the aluminium plates. The distance from the source to the each detector is equal to $35 \mathrm{~cm}$. The source with the plates was shifted above the line that connects the centers of the two detectors, and additionally a $10 \mathrm{~cm}$ thick lead block was inserted midway between the two detectors. This insertion ensured that photons did not Compton scatter from one detector into the other. For this configuration a simple relativistic kinematics calculation gives the minimum total energy for a positron annihilating in flight [6]:

$$
E_{\text {min }}=\frac{4 m_{0} c^{2}}{1-\cos (\theta)}-m_{0} c^{2},
$$

where $\theta$ is the angle between the source and the middle of the detectors (Fig. 2). Selecting the angle, we can select the minimal kinetic energy of positrons observed in our setup.

In Fig. 3a we present the results of detection of two photons which occur during the AiF process for $\theta=120^{\circ}$ using the setup. For comparison the measurement for $\theta=180^{\circ}$ i.e. AaR is also presented in Fig. 3b. In both cases the observed curves represent the prompt peaks (described well by Gaussian curve), because both annihilating photons reach detectors at the same time. For better comparison, the counts in both peaks were normalized to the measurement time. It is apparent that the prompt peak for the AaR process is much pronounced, due to the fact that process of thermalization is very efficient and except a small number all positrons finally annihilate from the thermal state. The prompt peak detected for the AiF process is much less pronounced. Additionally, the peak for the AaR is much narrower than for the AiF. Using the delay unit we performed the time calibration of our setup (for more details see Ref. [7]) which was 6.059 ps/channel. From that, the full width at the half maximum (FWHM) for the 


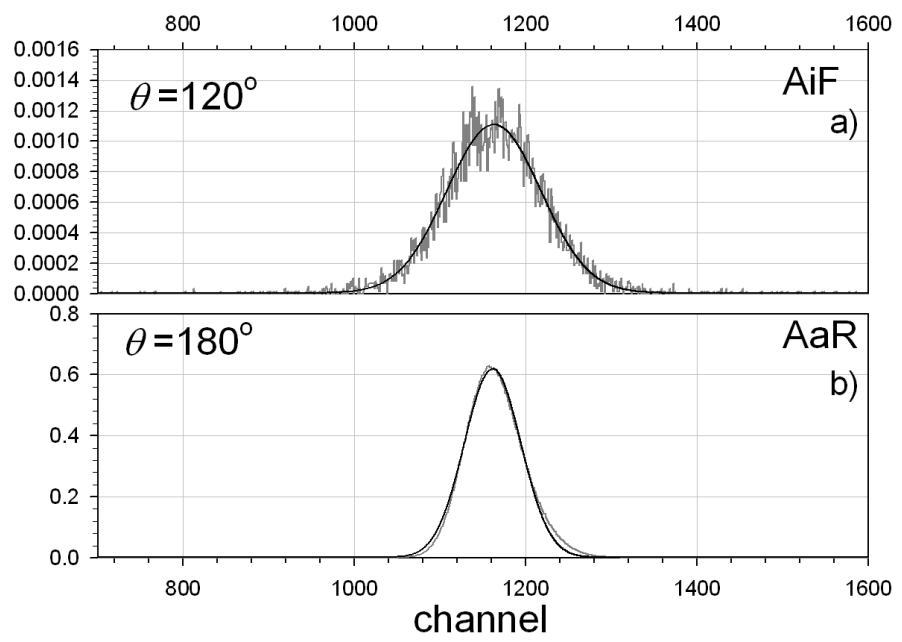

Fig. 3. The prompt peak (a) measured when the angle $\theta$ between the directions of emitted photons is equal to $120^{\circ}$; it corresponds to the AiF process. In (b), for comparison the prompt peak is presented when $\theta=180^{\circ}$ i.e., when AaR process was detected. The peaks were normalized to time of measurement. Solid black lines represent the Gaussian curve fitted to the prompt peaks.

AaR process is 483 ps and for AiF is almost doubled 820 ps. The reason of such differences is due to the fact that energy range fixed in both detector branches is very wide. This is not suitable for CFD units. For the AiF process energy of photons vary within the wide range, thus the pulses, which leave the anodes of photomultipliers, have wide range of amplitude. This is opposition to the AaR process where both photons coming to the detectors have similar energy close to $511 \mathrm{keV}$, thus the output pulses have much narrower range of amplitude. The latter gives less random walk of the standard NIM pulses produced by CFD. In a typical positron lifetime spectrometer the prompt peak is even more narrow, less than $300 \mathrm{ps}$, but for that case the energy range is much better tuned, because the energy of the detected photons is well defined in both branches.

Nevertheless, the FWHM of both peaks is not so significant as the total area under the peaks per unit time. This quantity is proportional to the number of positrons which annihilating produce photons, the angle between these photons is $\theta$. In the new experiment we measured the total area of the prompt peak per unit time (hereafter referred to as the intensity) as the function of the angle between the detectors keeping the source in the same position (Fig. 2). It is clear that decreasing the angle, we can select positrons, which annihilate with higher energy. This is possible because the isotope source emits positrons having a continuous energy distribution.

Figure 4 presents the dependence obtained. In this figure two separated regions are well visible. At the angle $\theta$ smaller than $180^{\circ}$ and more than $172^{\circ}$ (the 
shaded area), where the intensity is much higher, the region of the AaR process is extended. The width of this region can be confusing, because in the typical angular measurements of the annihilating photons (ACAR) the width of the AaR region is smaller than $2^{\circ}$. Nevertheless, in our experiment the angular resolution is very poor, i.e. about $4^{\circ}$. The characteristic parabolic decrease in the intensity with the decrease in the angle is well recognized from 1D ACAR experiments.

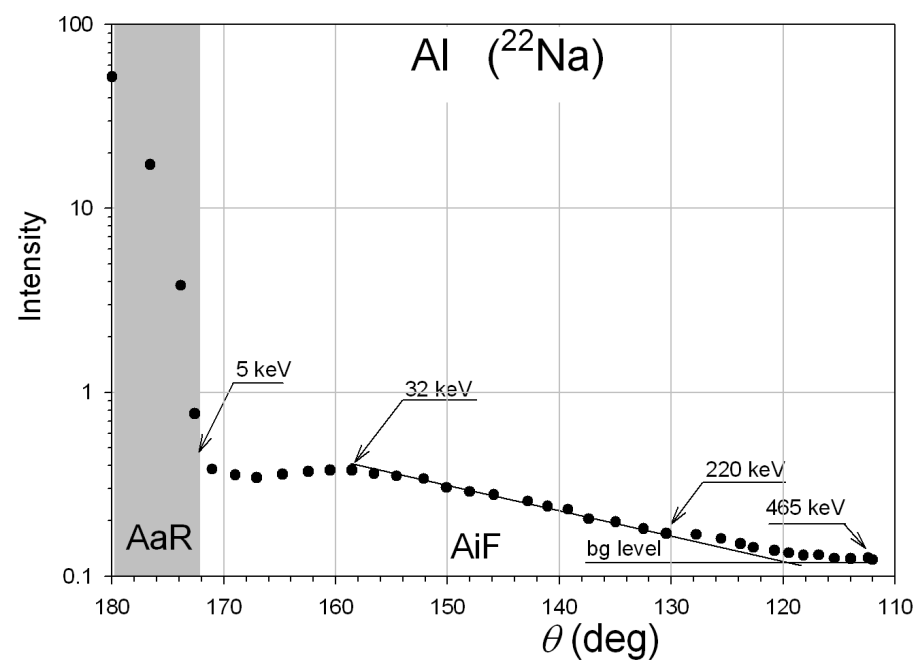

Fig. 4. The dependence of the area under the prompt peak per unit time on the angle $\theta$ between the directions of emitted annihilating photons. The measurements were performed using the setup presented in Fig. 2. The positrons emitted from the ${ }^{22} \mathrm{Na}$ isotope were stopped in two aluminum plates.

The AiF process contributes in the region below $170^{\circ}$, (Fig. 4). At this stage, we state that this region reflects the energy distribution of positrons emitted from the ${ }^{22} \mathrm{Na}$ nucleus, the total cross-section of annihilation process, and the positron stopping power. All these quantities strongly depend on the positron energy but in the different way. However, only the energy distribution does not behave as a monotonic function and exhibits onset, maximum and the end point. Up to the angle of $158^{\circ}$ the intensity slightly increases and reaches a maximum at $162^{\circ}$, it corresponds to the energy of $32 \mathrm{keV}$. This can be interpreted as the onset of the positron energy spectrum. After that, the intensity exponentially decreases to the angle of $128^{\circ}$ where small deviation is observed. At this angle the energy of positrons is close to $220 \mathrm{keV}$ which well corresponds to the maximum in the energy distribution of positrons emitted from the source which is equal to $210 \mathrm{keV}$ [8]. Then the intensity again decreases to the background level, which is reached at the angle of $114^{\circ}$. This corresponds to the positron energy of $467 \mathrm{keV}$. It is lower than the end point of the positrons distribution emitted form the source i.e. $540 \mathrm{keV}$. Nevertheless, at these angles, the energy selection is very poor, for the 
latter energy the corresponding angle is only $6^{\circ}$ lower i.e. $108^{\circ}$. The decrease in the intensity in the AiF region is well understood because the cross-section for annihilation decreases with the increase in the positron energy.

From Fig. 4 we can estimate the probability of the AiF process or the fraction of positrons which annihilate in this process. The ratio of the area under the region where the AiF process contributes to the total area is that probability and for $\mathrm{Al}$ we obtained that this quantity is equal to $c a .4 .76 \%$. Theoretical calculations performed for aluminum pointed out that this value is equal to $0.98 \%$ [3]. These discrepancy reflects the experimental and theoretical difficulties for detecting the AiF process.

At the angle smaller than $115^{\circ}$ the background level was reached (Fig. 3). In the case of ${ }^{22} \mathrm{Na}$ source, the main contribution to the background, comes from the coincidence of $511 \mathrm{keV}$ annihilation photons with $1275 \mathrm{keV}$ gamma. The latter is detected as the wide range Compton spectrum. Nevertheless, because there is no spatial correlation between the direction of emission of two 1275 gamma and annihilation photons, thus we argue that these events give constant background level over the whole angle range. We estimated that about 0.11 counts/s of intensity comes from these events. Other background sources: like cosmic ray or natural isotopes contribute about 0.01 counts/s to the background level. Our future measurements, where ${ }^{22} \mathrm{Na}$ source was replaced by ${ }^{68} \mathrm{Ge} \backslash{ }^{68} \mathrm{Ga}$ source, confirmed these arguments. Nevertheless, the answer to the question if the background level is a constant in the whole range of measured angles requires more accurate measurements, which we intend to perform in future.

\section{Conclusion}

The conclusions of the present work can be summarized as follows. The new experimental setup which is a modification of the conventional positron lifetime spectrometer allows us to map the AiF and AaR processes. They were well separated. In the region of the $\mathrm{AiF}$ we were able to recognize the characteristic features of the positron energy distribution emitted from the ${ }^{22} \mathrm{Na}$. As we expected, a decrease in the intensity of this process with the increase in the positron energy was also observed. We believe that the presented setup allows to get to know more about the AiF process in condensed matter.

\section{References}

[1] P.A.M. Dirac, Proc. Cambridge Philos. Soc. 26, 361 (1930).

[2] R. Krause-Rehberg, H.S. Leipner, Positron Annihilation in Semiconductors: Defect Studies, Springer, Berlin 1998.

[3] J. Dryzek, in: Proc. 33th PSPA, Turawa (Poland) 2001, Ed. K. Jerie, Institute of Physics, University of Opole, Institute of Experimental Physics, University of Wrocław, Opole 2001, p. 33.

[4] M.Z. Akrawy et al., Phys. Lett B 257, 531 (1991). 
[5] M.H. Weber, A.W. Hunt, J.A. Golovchenko, K.G. Lynn, Phys. Rev. Lett. 83, 4658 (1999).

[6] J. Dryzek, D. Singleton, T. Suzuki, R. Yu, Am. J. Phys. 74, 49 (2006).

[7] J. Dryzek, D. Singleton, Am. J. Phys. 75 713, 07 (20.)

[8] B.T. Wright, Phys. Rev. 90, 159 (1953). 